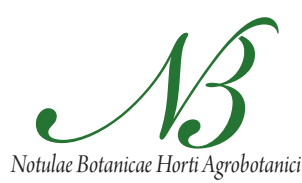

Cluj-Napoca

\title{
New Markers for Potato Late Blight Resistance and Susceptibility Using FTIR Spectroscopy
}

\author{
Abdelmoumen TAOUTAOU*, Carmen SOCACIU, Doru PAMFIL, \\ Florinela FETEA, Erika BALAZS, Constantin BOTEZ \\ University of Agricultural Sciences and Veterinary Medicine Cluj-Napoca, 3-5 Calea Manastur, \\ 400372, Cluj-Napoca, Romania; tamoumen@gmail.com ("corresponding author)
}

\begin{abstract}
The potato (Solanum tuberosum) is the $3^{\text {rd }}$ most important crop worldwide, and Phytophthora infestans is the most devastating pathogen to potato crops. In this study, it has identified markers for resistance and susceptibility to late blight using potato genotypes that differ in their resistance/susceptibility to Phytophthora infestans. Using Fourier transform infrared spectroscopy, many absorbance bands have been identified as specific to resistant potato plants, and others were specific to susceptible potato plants. For each case (resistance/susceptibility), three bands were identified: $941-1180 \mathrm{~cm}^{-1}, 1336-1483 \mathrm{~cm}^{-1}$, and $1483-1703 \mathrm{~cm}^{-1}$ and $1056-1294 \mathrm{~cm}^{-1}, 1442-$ $1585 \mathrm{~cm}^{-1}$, and 1585-1832 $\mathrm{cm}^{-1}$, respectively. As it is a simple, rapid, and inexpensive technology, FTIR (Fourier Transform Infrared) spectroscopy offers an excellent opportunity for studying potato resistance/susceptibility to late blight.
\end{abstract}

Keywords: FTIR, markers, MAS, Phytophthora infestans, resistance, Solanum tuberosum, susceptibility

\section{Introduction}

Late blight is the major disease of potato crops and is also important in tomato and other Solanaceae species. The disease is caused by the oomycete Phytophthora infestans, which is a heterothallic hemibiotrophic pathogen. When $S$. tuberosum is the host, the asexual life cycle of $P$. infestans can be completed rapidly, with the production of a massive number of sporangia that are readily dispersed. Such a process explains why entire fields can be transformed from slightly diseased to almost completely destroyed within only a few days (Flor, 2008).

$P$. infestans uses a multitude of effectors to produce a successful infection. As defined by Kamoun (2006), effectors are molecules that manipulate the host cell structure and function, thereby facilitating infection (infection factors or toxin) and/or triggering defence responses (avirulence factors or elicitors).

Late blight control is mainly based on fungicides. However, potato resistance plays an important role in controlling late blight and also in optimising fungicide protection (Sedláková et al., 2011). Potato breeding for late blight resistance has been based on the introgression of resistance $(R)$ genes from the related wild species $S$. demissum. To date, $11 R$ genes have been introduced in different potato cultivars around the world; however, $P$. infestans was able to overcome the resistance conferred. Resistance breeding is also based on resistance genes from other wild species, such as $S$. bulbocastanum and $S$. phure; very recently, gene pyramiding (gene stacking) has been used to introduce several (at least 2) $R$ genes from different Solanum species into varieties of cultivated potatoes.

Marker-assisted selection (MAS) offers many advantages to breeders: it reduces the time necessary for testing the plant materials and the effort and money required to accomplish a successful gene introgression in the genotype of interest. In general, MAS uses molecular markers as tools for the identification of resistance genes and resistant materials. However, molecular techniques remain expensive, time consuming and not all laboratories can afford them. Therefore, the development of a new simple, inexpensive and rapid procedure for selection is vitally important.

Fourier Transform Infrared (FTIR) spectroscopy is a simple method, and its applications can be easily and quickly used. Furthermore, FTIR can be used as a nondestructive technique and is a promising alternative to the molecular techniques used in MAS. FTIR has not been used thus far in MAS, and FTIR has seldom been utilised to study plant pathology (Bertoluzza et al., 1999; Stewart et al., 1994; Taoutaou et al., 2010).

In this work, it has attempted to identify specific markers for potato resistance/susceptibility to the late blight agent $P$. infestans using a quick and inexpensive technique, FTIR spectroscopy. This report is a follow up to a previous study regarding the application of FTIR to studying interactions in the $P$. infestans-S. tuberosum pathosystem (Taoutaou et al., 2010). 


\section{Materials and methods}

\section{Plant material}

The potato plants used in this study were divided into two groups: resistant and susceptible. The first group was represented by Solanum demissum (dms), Solanum bulbocastanum (blb), and 2 accessions of Solanum tuberosum ('R4', a potato plant with the resistance gene $R 4$, and ' 21 ', which has three $R$ genes $R 2, R 3$, and $R 4$ ). The susceptible potato genotypes are all $S$. tuberosum cultivars: the cultivars 'Desiree' and 'Bintje', which lack resistance genes, and four accessions with different resistance genes, $R 1, R 2$, $R 3$, and $R 5$, named for the gene they possess.

\section{Pathogen}

Two isolates of $P$. infestans have been used to inoculate the potato genotypes: A2.2 (NL08009) and A2.3 (88133). The $P$. infestans isolates were kindly provided by WG Flier, GBM van den Bosch and GJT Kessel from Plant Research International BV.

\section{Inoculation}

The inocula preparation and leaf inoculation were performed according to Cip manual (1997) using the detached leaf test.

\section{FTIR procedure}

In the fifth day post-inoculation, the leaves were ground into a fine powder using a mortar and pestle with liquid nitrogen and then transferred to a $2 \mathrm{ml}$ Eppendorf tube. After adding $1 \mathrm{ml}$ of $70 \%$ methanol, the mixture was sonicated for 15 minutes and then centrifuged. A $100 \mu \mathrm{l}$ aliquot of the supernatant was used for the analysis. The infrared profile was determined using the Shimatzu Prestige 2, Apodization: Happ-Genzel spectrophotometer in the wavelength range of $4000-500 \mathrm{~cm}^{-1}$.

\section{Results and discussion}

As expected, the pathogen isolate was able to infest the susceptible potato plants. The reactions to inoculation were different for the resistant potato genotypes: a hypersensitive reaction was detected for genotype ' 21 ', but it has observed no hypersensitivity or other symptoms for ' $\mathrm{R} 4$ '. The symptoms are shown in Fig. 1.

The FTIR spectra for the potato genotypes are shown in Fig. 2 for the susceptible genotypes and in Fig. 3 for the resistant genotypes. Overall, the FTIR spectra for the susceptible plants exhibit the same shape (Fig. 2); however, differences are detectable when the spectra are studied in detail and by specific intervals.

These results were also found for the resistant genotypes, with the exception that the 'R4' spectra of the control (non-inoculated) and inoculated plants can be nearly superposed (Fig. 3).

When the spectra of the resistant and susceptible genotypes are combined, specific bands for each group are easily identifiable (Fig. 4), with the following major bands: $941-1180 \mathrm{~cm}^{-1}, 1336-1483 \mathrm{~cm}^{-1}$, and $1483-1703 \mathrm{~cm}^{-1}$ for the resistant genotypes and 1056-1294 $\mathrm{cm}^{-1}, 1442-1585$ $\mathrm{cm}^{-1}, 1585-1832 \mathrm{~cm}^{-1}$ for the susceptible genotypes (Fig. 4).

In addition to these major specific bands, it has also examined many smaller bands and/or peaks that were specific to the resistant or susceptible genotypes. Bands at 631-637 and $683-770 \mathrm{~cm}^{-1}$ were detected in inoculated 'R4' and $S$. demissum, all of the treatments of ' 21 ', and the control and infected $S$. bulbocastanum. The band at $949-1016 \mathrm{~cm}^{-1}$ was also specific to the resistant potato genotypes, with the exception of the peak at $691 \mathrm{~cm}^{-1}$, which was detected only in the uninfected 'Bintje'. Near to the last band (the band at $949-1016 \mathrm{~cm}^{-1)}$, the susceptible plants had absorbances at $1072-1076 \mathrm{~cm}^{-1}$, whereas the resistant plants had absor-
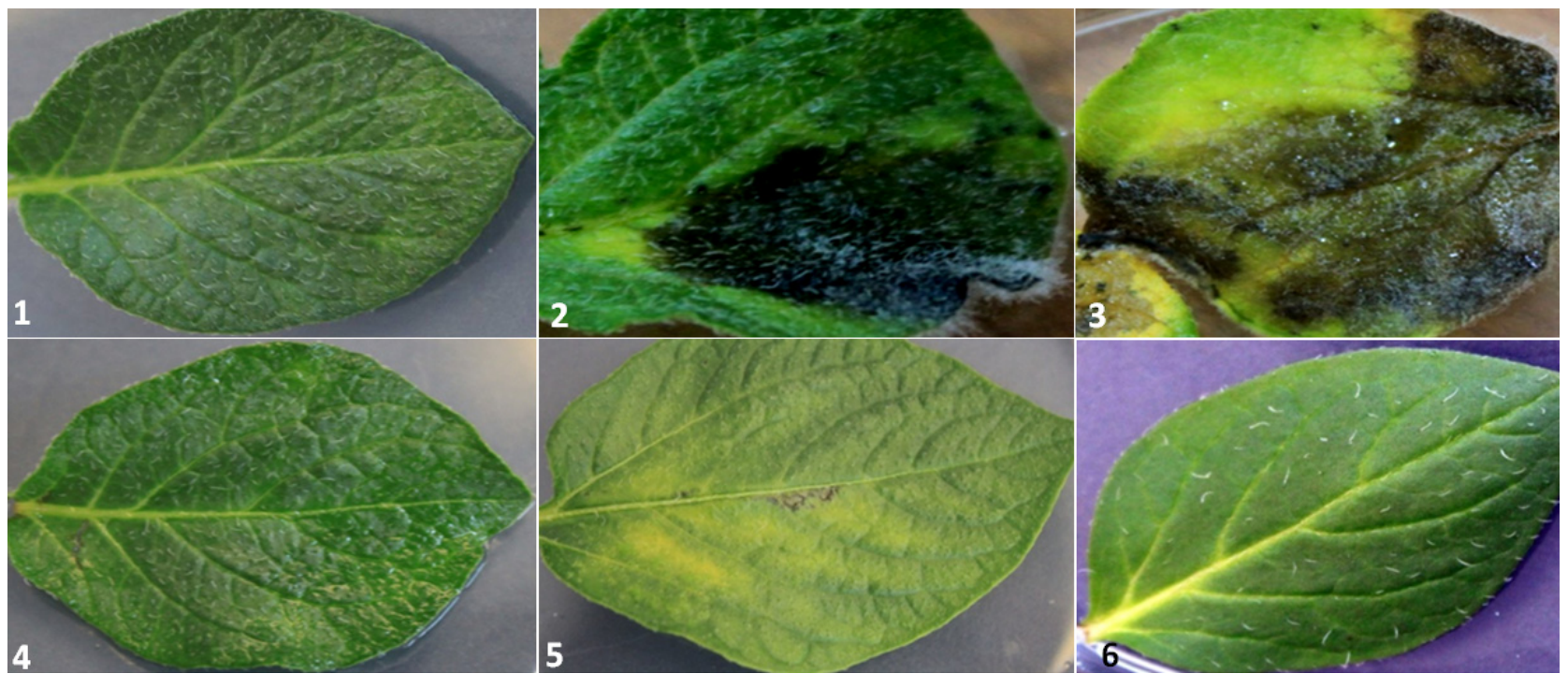

Fig. 1. Reactions of different potato genotypes to the inoculation with $P$. infestans and the symptoms of the susceptible genotypes. 1-Control; 2-'R2'; 3-'Bintje'; 4-'R4'; 5-'21'; 6-S. demissum. On 'R2' and 'Bintje', the mycelia can easily be observed on the leaves; '21' reacted with a hypersensitive response, whereas no reactions were detected for 'R4' and $S$. demissum 
152

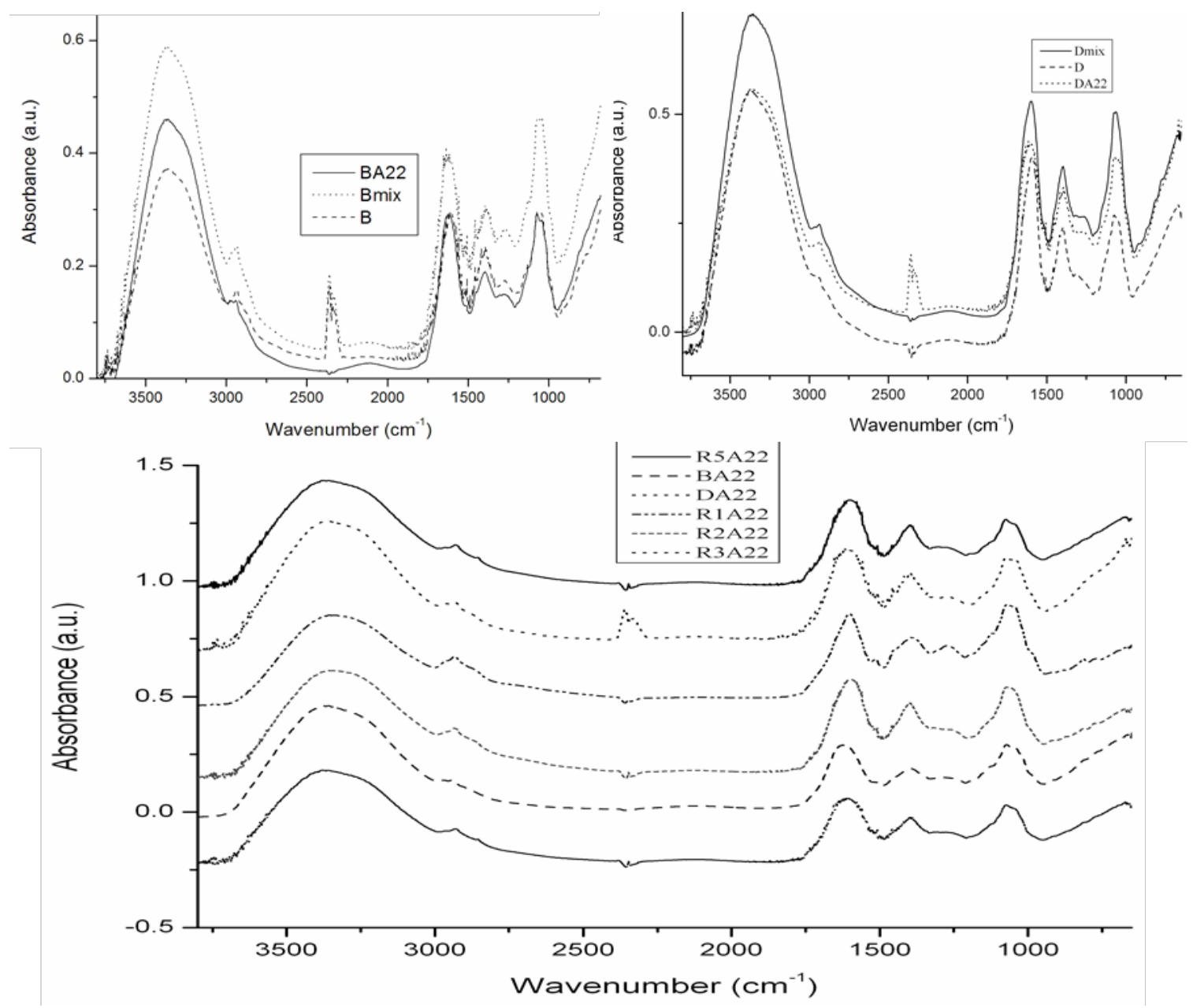

Fig. 2. FTIR spectra of susceptible potato genotypes (inoculated 'Bintje' and 'Desiree' with A22 and a mix of A22+A23) and the control and a compilation of the spectra of different inoculated genotypes with A22. The spectra have the same shape with minor differences. The major bands are common between all genotypes; however, the absorption intensities differ in the function of the genotype
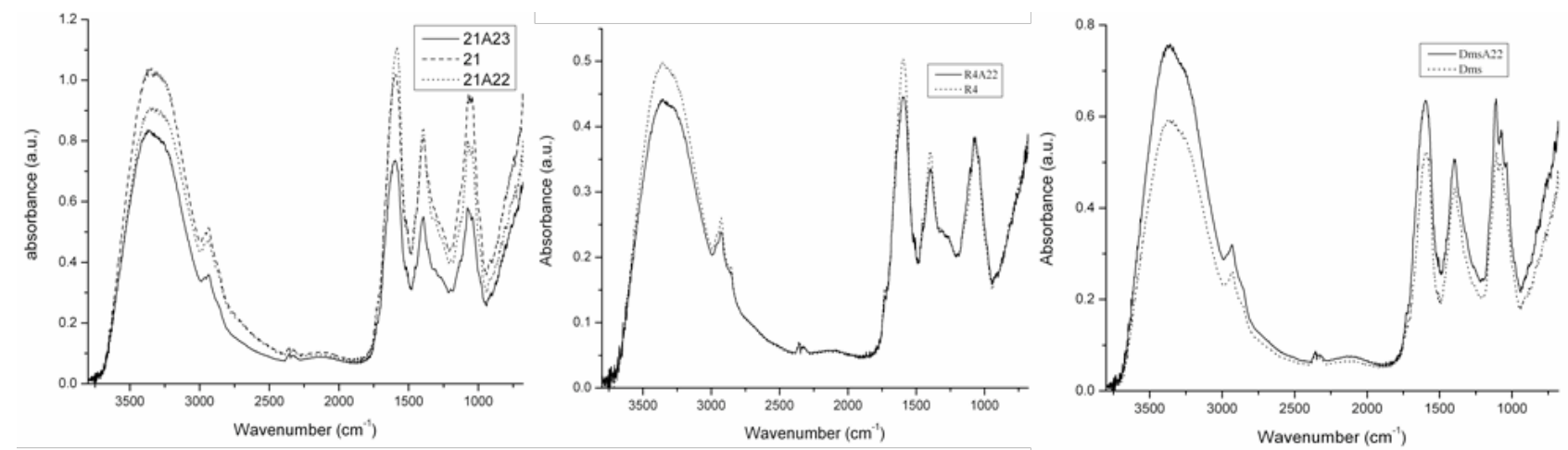

Fig. 3. FTIR spectra of different resistant genotypes with different treatments (control and inoculated). The spectra are approximately superposed in the case of 'R4'; in the other cases, only the absorption intensities differ

bances at $1078-1109 \mathrm{~cm}^{-1}$. The $1196-1200 \mathrm{~cm}^{-1}$ band was common to most of the plants, regardless of their resistance/susceptibility status. An interesting band specific to S. tuberosum was found at $1396-1400 \mathrm{~cm}^{-1}$ and was not detected in $S$. demissum or $S$. bulbocastanum. Another band specific for resistant plants is the band at $1238-1265 \mathrm{~cm}^{-1}$. Whereas the resistant $S$. tuberosum and inoculated R1 had absorbances at $1506 \mathrm{~cm}^{-1}$, the susceptible plants showed absorbances in the $1524-1553 \mathrm{~cm}^{-1}$ and $1558-1572 \mathrm{~cm}^{-1}$ intervals. Other resistance bands have been found: 1576$1589 \mathrm{~cm}^{-1}, 2291-2313 \mathrm{~cm}^{-1}, 2590-2932 \mathrm{~cm}^{-1}$ (for the last interval, some exceptions were found for the control R1 at $2590 \mathrm{~cm}^{-1}$ and the infected R3 at $2617 \mathrm{~cm}^{-1}$ and 2855 $\left.\mathrm{cm}^{-1}\right), 3015-3262 \mathrm{~cm}^{-1}$, and $3271-3275 \mathrm{~cm}^{-1}$. Other sus- 


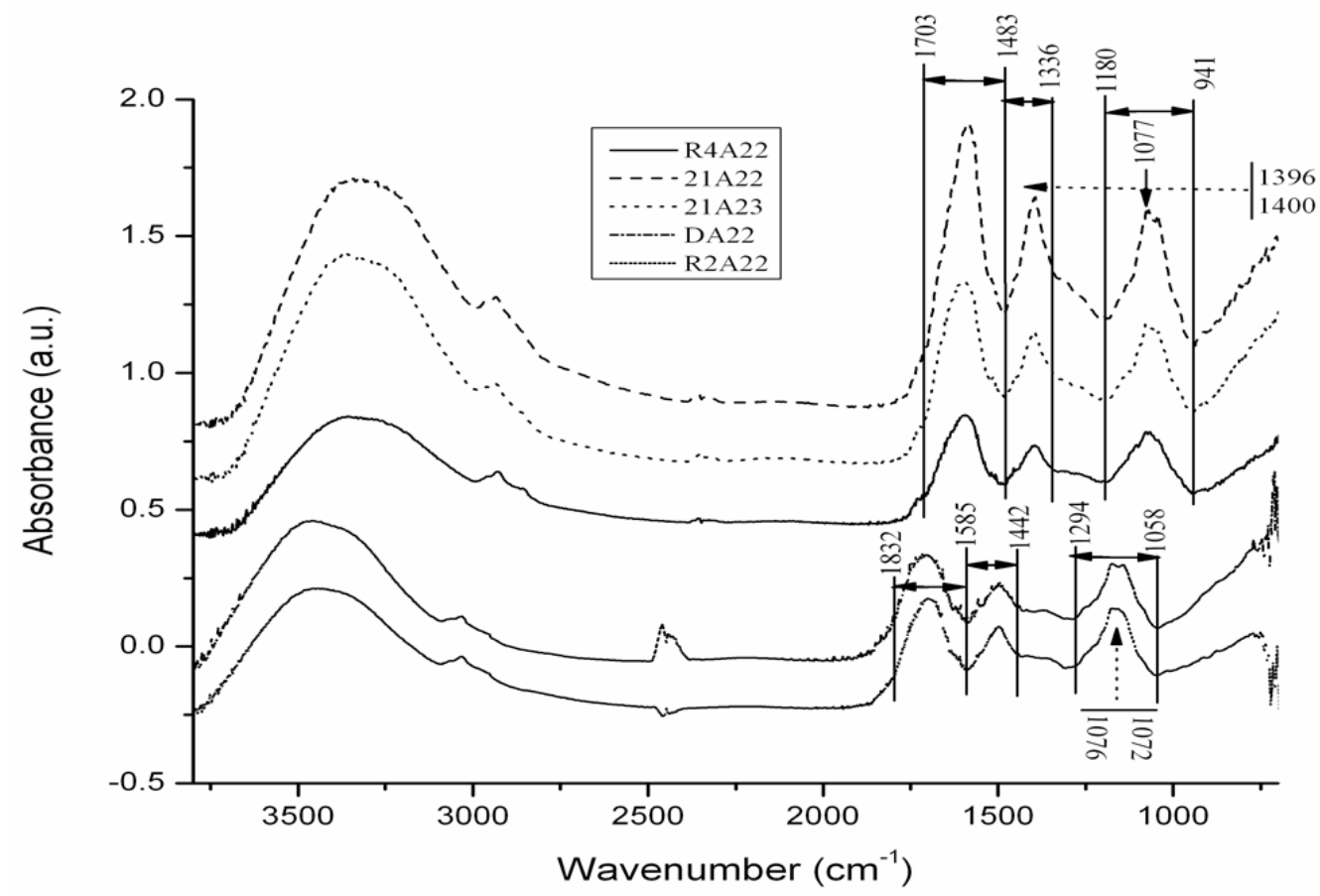

Fig. 4. FTIR spectra with characteristic bands of resistant and susceptible potato plants to the late blight agent. All the spectra show the same behavior; however, for susceptible plants, there is a shift to a longer wavelength of the bands for the susceptible genotypes. Resistant: $941-1180 \mathrm{~cm}^{-1}$, susceptible: 1056 $1294 \mathrm{~cm}^{-1}$; resistant: $1336-1483 \mathrm{~cm}^{-1}$, susceptible: $1442-1585 \mathrm{~cm}^{-1}$; resistant: $1483-1703 \mathrm{~cm}^{-1}$, susceptible: $1585-1832 \mathrm{~cm}^{-1}$

ceptibility bands have been detected at $1603-1630 \mathrm{~cm}^{-1}$, $1969-2041 \mathrm{~cm}^{-1}, 2062-2152 \mathrm{~cm}^{-1}$, and $2270-2284 \mathrm{~cm}^{-1}$.

FTIR is a simple, easy and rapid technique that provides a large amount of information for the study of plant pathology. For single-celled organisms, such as bacteria and some fungi, FTIR measures the total composition of the cell in a non-destructive manner, producing an IR spectrum with bands for all of the cellular components (e.g., membranes, proteins, nucleic acids) (Oust et al., 2004). However, the situation is somewhat different in the case of plants. Compounds, such as matrix heteropolymers, pectins and hemicelluloses, phenolic oxidation products, dyes, alcohols, terpenes, tannins, additional waxes, oils, resins, salt crystals, and mucilage, which are often excreted on the plant cell surface, create difficulty when using nondestructive spectroscopic methods for the evaluation of the plant status (Ivanova and Singh, 2003). However, even with these difficulties, FTIR is still a simple, rapid, inexpensive and reagent-free technology.

An FTIR spectrum can be divided into five zones. The first is called the fingerprint zone, in the $600-900 \mathrm{~cm}^{-1}$ range. The second is situated in the interval of 900-1200 $\mathrm{cm}^{-1}$ and is specific for polysaccharides and carbohydrates. The third zone in the interval $1200-1500 \mathrm{~cm}^{-1}$ is a mixed region detecting proteins, fatty acids, DNA, RNA, and groups with phosphorus molecules. The fourth zone, at $1500-1800 \mathrm{~cm}^{-1}$, is characteristic of amides and proteins (e.g., amide I, amide II, proteins, peptides) and phenols and polyphenols. The fifth and last region is the fatty acid zone in the interval of $2800-3200 \mathrm{~cm}^{-1}$.
For the resistant genotypes, the specific markers are situated within the polysaccharide and carbohydrate zones, the mixed zone and the amide and phenol regions. In the case of susceptible plants, the first specific band is part of the polysaccharide and mixed zones, and the second band is contained within the mixed region but also within the amide and phenolics zones. The third band is situated in the amide/protein and phenolics regions. $\mathrm{Nev}$ ertheless there is a delay of bands in the case of the susceptible genotypes. This delay indicates a low concentration of the metabolites and molecules implicated in resistance, which are present in the resistant genotypes. The susceptible genotypes synthesise other molecules from the same group, but the molecules have no effect on the outcome of the potato-Phytophthora interaction. Metlitsky and Ozheretskovskaya (in Dyakov and Dzhavakhiya, 2007) found that the synthesis of terpenoids in resistant and susceptible potato varieties proceeds in a similar manner up to the stage of $\mathrm{C} 15$ farnesyl pyrophosphate production. After this point, two molecules of farnesyl pyrophosphate condense (catalysed by squalene synthetase) in susceptible varieties, with the production of $\mathrm{C} 30$ squalene from which phytosterols are produced. In contrast, under the effect of another enzyme (cyclase) in the resistant varieties, the farnesyl pyrophosphate molecules close, forming two rings and producing bicyclic sesquiterpenes, which are highly toxic phytoalexins. Thus, instead of providing a required nutrient, the plant furnishes the parasite with a toxin (Dyakov and Dzhavakhiya, 2007). 
154

The small band at $631-637 \mathrm{~cm}^{-1}$ is important and could be considered as a marker for resistance, especially because it is situated in the fingerprint zone. This band was detected all of the resistant genotypes, both in the control and inoculated leaves.

In addition, these bands and peaks could be considered as markers for compatible Solanum spp.-P. infestans interactions in the case of the susceptible genotypes and as markers for incompatible interactions in the case of the resistant genotypes.

If the advantages of MAS could be combined with the FTIR technique, it would offer significant reductions in cost and time to breeders and researchers. MAS is currently used for gene pyramiding and for pyramided QTLs (Ashikari and and Matsuoka, 2006). Gene pyramiding is the accumulation of resistance genes (in this case) into a single genotype or cultivar (Tan et al., 2010), such as genotype ' 21 ' in the present study.

In this study, it has demonstrated that FTIR can be used in plant pathology research. FTIR is not yet popular among plant pathologists; however, the potential opportunities offered by this technique are manifold (Bertoluzza et al., 1999; Ivanova and Singh, 2003; Stewart et al., 1994; Taoutaou et al., 2010). FTIR is used also in many fields similar to plant pathology, such as mycology and human medicine. In their review, Santos et al. (2010) describe FTIR as a powerful technique for the identification and characterisation of filamentous fungi and yeast. Using synchrotron FTIR spectromicroscopy, Jikline et al. (2008) were able to study the chemical composition of filamentous fungi at the single-cell level.

\section{Conclusions}

The bands identified in this study could be considered as markers for resistance $\left(941-1180 \mathrm{~cm}^{-1}, 1336-1483 \mathrm{~cm}^{-1}\right.$, and $1483-1703 \mathrm{~cm}^{-1}$ ) or at least as markers for an incompatible Solanum spp.-Phytophthora infestans interaction. Conversely, the $1056-1294 \mathrm{~cm}^{-1}, 1442-1585 \mathrm{~cm}^{-1}$, and $1585-1832 \mathrm{~cm}^{-1}$ bands could be considered as markers for susceptibility in this pathosystem or at least as markers for a compatible interaction.

Despite its lower popularity among plant pathologists, FTIR spectroscopy offers an important opportunity for studying potato late blight resistance and/or susceptibility, as it is a simple, rapid and inexpensive technique.

\section{Acknowledgements}

This project is funded by the Ministry of Education and Research, Romania, within the framework of the PN II program, project no. $1050 / 1009$, and code CNCSIS 1496. This work has also benefited from financial support through the 2010 POSDRU/89/1.5/S/52432 project, "Organizing Tthe National Interest Postdoctoral School of Applied Biotechnologies with Impact On Romanian Bioeconomy", a project co-financed by the European So- cial Fund through the Sectoral Operational Programme Human Resources Development 2007-2013.

\section{References}

Ashikari M, Matsuoka M (2006). Identification, isolation and pyramiding of quantitative trait loci for rice breeding. Trends Plant Sci 11:344-350.

Bertoluzza A, Bottura G, Lucchi P, Marchetti L, Zechini d'Aulerio A (1999). Molecular monitoring of horse chestnut leaves affected with biotic and abiotic disorders. J Plant Pathol 81:89-94.

CIP (1997). Laboratory manual for $P$. infestans work at CIPQUITO. Cip, Peru, 36 p.

Dyakov YT, Dzhavakhiya VG (2007). Biochemical resistance factors, 164-179 p. In: Dyakov YT, Dzhavakhiya VG, Korpela $\mathrm{T}$ (Eds.). Comprehensive and molecular phytopathology. Elsevier, Amsterdam, The Netherlands.

Flor W (2008). Phytophthora infestans: The plant (and $R$ gene) destroyer. Molec Plant Pathol 9:385-402.

Ivanova DG, Singh BR (2003). Nondestructive FTIR monitoring of leaf senescence and elicitin-induced changes in plant leaves. Biopolymers (Biospectroscopy) 72:79-85.

Jikline K, Gough KM, Julian R, Kaminsky SGW (2008). A sensitive method for examining whole-cell biochemical composition in single cells of filamentous fungi using synchrotron FTIR spectromicroscopy. J Inorg Biochem 102:540-546.

Kamoun S (2006). A catalogue of the effector secretome of plant pathogenic oomycetes. Annu Rev Phytopathol 44: 41-60.

Oust A, Moretro T, Kirschner C, Narvhus JA, Kohler A (2004). FTIR spectroscopy for identification of closely related lactobacilli. J Microbiol Methods 59:149-162.

Santos C, Fraga ME, Kozakiewicz Z, Lima N (2010). Fourier transform infrared as a powerful technique for the identification and characterization of filamentous fungi and yeast. Res Microbiol 161:168-175.

Sedláková V, Dejmalová J, Hausvater E, Sedlák P, Doležal P, Mazáková J (2011). Effect of Phytophthora infestans on potato yield in dependence on variety characteristics and fungicide control. Plant Soil Environ 57:486-491.

Stewart D, Lyon GD, Tucker EJB (1994). A Fourier-transform infrared spectroscopic and microscopic study of the infection of potato tubers by Erwinia carotovora ssp. carotovora in aerobic and anaerobic conditions. J Sci Food Agric 66:145154.

Tan MYA, Hutten RCB, Visser RGF, van Eck HJ (2010). The effect of pyramiding Phytophthora infestans resistance genes $R_{P_{i}-\text { medl }}$ and $R_{P_{i}-b e r}$ in potato. Theor Appl Genet 121:117-125.

Taoutaou A, Socaciu C, Pamfil D, Fetea F, Balazs E, Botez C, Chis A, Briciu D, Briciu A (2010). Fourrier transformed infrared spectroscopy applied for studying compatible interaction in the pathosystem Phytophthora infestans-Solanum tuberosum. Not Bot Horti Agrobo 38:69-75. 